\title{
HODGE THEORY OF SINGULAR ALGEBRAIC CURVES
}

\author{
MASAYOSHI NAGASE
}

(Communicated by Frederick R. Cohen)

\begin{abstract}
The spectrum of the Laplacian and the structure of the $L^{2}$-cohomology are studied on a singular algebraic curve with its singularity removed, which is hence an incomplete Kähler manifold.
\end{abstract}

\section{Statements of the Results}

Let $C$ be a complex algebraic curve embedded in the projective space $\mathbf{P}^{N}(\mathbf{C})$ and $S$ be its singularity set. Put $C^{*}=C-S$ and denote by $g$ the restriction (to $C^{*}$ ) of the Fubini-Study metric of $\mathbf{P}^{N}(\mathbf{C})$. The author wishes to develop the global analysis (or, the spectral geometry) on the incomplete Kähler manifold $C^{*}=C^{*}(g)$.

First let us show that $C^{*}$ is quasi-isometric to a real 2-dimensional incomplete Kähler manifold with cone-like singularities in the sense of $\mathbf{J}$. Cheeger ([1], [3]). Take a nonsingular model $\pi: \widetilde{C} \rightarrow C$ and set $\widetilde{C}^{*}=\widetilde{C}-\pi^{-1}(S)$. Then, since the map $\pi$ gives an isometry $\widetilde{C}^{*}\left(\pi^{*} g\right) \rightarrow C(g)$, let us investigate $\pi^{*} g$ near $\pi^{-1}(S)$. By a standard argument (see, for example, [12, Lemma 1.6.1]), for a point $p \in \pi^{-1}(S)$, there exists a local coordinate neighborhood $(U, u)$ with $u=0$ at $p$ and the affine coordinates $\left(z_{1}, \ldots, z_{N}\right)$ of $\mathbf{P}^{N}(\mathbf{C})$ with $\left(z_{1}, \ldots, z_{N}\right)=(0, \ldots, 0)$ at $\pi(p)$ such that $z_{1}(u)=u^{m}, z_{2}(u)=$ $u^{m_{2}} f_{2}(u), \ldots, z_{l}(u)=u^{m_{1}} f_{l}(u), z_{l+1}(u)=0, \ldots, z_{N}(u)=0$, where $1<$ $m<m_{2}<\cdots<m_{l}$ and moreover $f_{j}(0)=1$. The number $m$ is the multiplicity of $\pi(p)$ on the irreducible component determined by $p$. Now, since the Fubini-Study metric and the metric $\sum_{i=1}^{N} d z_{i} \otimes d \bar{z}_{i}$ are quasi-isometric on a neighborhood of $\pi(p)$, obviously on $U-p$ the two metrics $\pi^{*} g$ and $\pi^{*}\left(\sum_{i=1}^{N} d z_{i} \otimes d \bar{z}_{i}\right)$ are quasi-isometric (through the identity map). Moreover, since the square of the pointwise norm of a vector $a \partial / \partial u$ with respect to the second metric is equal to $\sum_{i}\left|\pi_{*}(a \partial / \partial u)\right|^{2}=|a|^{2} \sum\left|\partial z_{i} / \partial u\right|^{2}=$ $|a|^{2}\left\{m^{2}|u|^{2(m-1)}+\sum_{i>1}|u|^{2\left(m_{i}-1\right)}\left|m_{i} f_{i}+u f_{i}^{\prime}\right|^{2}\right\}$, the metric is quasi-isometric to $|u|^{2(m-1)} d u \otimes d \bar{u}$. Therefore, we have the following lemma.

Received by the editors May 27, 1988 and, in revised forms, December 16, 1988 and April 6, 1989.

1980 Mathematics Subject Classification (1985 Revision). Primary 58A14, 58C40. 
Lemma. On $U-p$, the metrics $\pi^{*} g$ and $|u|^{2(m-1)} d u \otimes d \bar{u}$ are quasi-isometric through the identity map.

Let us denote the circle of circumference $2 m \pi$ by $S_{m}^{1}$ and consider the metric cone on it, i.e. $C\left(S_{m}^{1}\right)="(0,1) \times S_{m}^{1}(\ni(r, \theta))$ with metric $d r^{2}+r^{2} d \theta^{2}$ ", which is the $m$-fold covering of the punctured unit disk with flat metric. Then obviously the lemma implies

Theorem ([4, p. 323], [5, Lemma 3.15]). The map $u \mapsto\left(|u|^{m}, m \arg u\right)$ induces the quasi-isometry from $\{u \in U-p|| u \mid<1\}$ with metric $\pi^{*} g$ to $C\left(S_{m}^{1}\right)$.

Hence $C^{*}$ is quasi-isometric to a manifold with cone-like singularities. And, according to [1]-[11], we obtain the following corollaries.

Let $\Lambda^{i}\left(C^{*}\right)$ and $L^{2} \Lambda^{i}\left(C^{*}\right)$ be the spaces consisting of smooth and squareintegrable (with respect to $g$ ) $i$-forms on $C^{*}$ respectively. Denote by $d_{i}$ and $\delta_{i}$ the exterior derivative $d$ and its formal adjoint $\delta$ with the following domains: $\operatorname{dom} d_{i}=\left\{\alpha \in \Lambda^{i}\left(C^{*}\right) \cap L^{2} \Lambda^{i} \mid d \alpha \in L^{2} \Lambda^{i+1}\right\}$, $\operatorname{dom} \delta_{i}=\left\{\alpha \in \Lambda^{i+1}\left(C^{*}\right) \cap\right.$ $\left.L^{2} \Lambda^{i+1} \mid \delta \alpha \in L^{2} \Lambda^{i}\right\}$. Moreover, letting $\Lambda_{c}^{i}\left(C^{*}\right)$ be the space of compactly supported smooth $i$-forms, we set $d_{c, i}=d \mid \Lambda_{c}^{i}\left(C^{*}\right)$ and $\delta_{c, i}=\delta \mid \Lambda_{c}^{i+1}\left(C^{*}\right)$. Their closures with respect to the operator norms are denoted by $\hat{d}_{i}, \hat{\delta}_{i}, \hat{d}_{c, i}, \hat{\delta}_{c, i}$. Now the first assertion is the following (see [1, Theorem 2.2]):

Corollary 1. $\hat{d}_{c, i}=\hat{d}_{i}, \hat{\delta}_{c, i}=\hat{\delta}_{i}$.

Let $\left(\right.$, ) be the global inner product (for forms on $C^{*}$ ) and let us denote by $\hat{d}_{i}^{*}$, etc. the dual operators (with respect to $\left.(),\right)$ of the intrinsic operators $\hat{d}_{i}$, etc. Then, since in general we have $\hat{d}_{i}=\hat{\delta}_{c, i}^{*}$ and $\hat{d}_{c, i}=\hat{\delta}_{i}^{*}$, the two equalities in Corollary 1 are equivalent. Moreover, they are equivalent to the equality $\hat{d}_{i}=\hat{\delta}_{i}^{*}$, which means that the Stokes' theorem in the $L^{2}$-sense $([1,(1.7)])$ holds for $C^{*}$.

Corollary 1 implies that the Laplacian $\Delta_{i}=\hat{\delta}_{i} \hat{d}_{i}+\hat{d}_{i-1} \hat{\delta}_{i-1}$ is self-adjoint with respect to $($,$) . (The Dirichlet and Neumann type Laplacians agree in this$ case and can be written as above without distinction.) The second assertion concerns the spectrum; see [3], [7].

Corollary 2. The spectrum of the Laplacian $\Delta_{i}$ consists only of eigenvalues $(0 \leq) \lambda_{0} \leq \lambda_{1} \leq \lambda_{2} \leq \ldots \uparrow \infty$ (with multiplicities) and there exists a constant $K>0$ such that

$$
\sum_{j=0}^{\infty} e^{-\lambda_{j} t} \leq K t^{-1}, \quad 0<t \leq t_{0} .
$$

As for the left hand side of (1.1), that is, the trace of the kernel of the heat operator $e^{-\Delta_{i} t}$, we have the following (weak) conjecture after the analogy of the nonsingular case, which must be proved by the same argument as in the nonsingular case. (However we will have to be very careful in making various estimates at each step.) Let us state it only for the case $i=0$. 
Conjecture. If $i=0$, then $\sum_{j=0}^{\infty} e^{-\lambda_{j} t} \sim \operatorname{Vol}(C) / 4 \pi t, t \downarrow 0$.

We will now discuss some properties of $\operatorname{Ker} \Delta_{i}$. Corollary 2 implies that Range $\hat{d}_{i-1}$ and Range $\hat{\delta}_{i}$ are both closed in $L^{2} \Lambda^{i}\left(C^{*}\right)$. Hence the Hodge decomposition can be written as follows: $L^{2} \Lambda^{i}\left(C^{*}\right)=\operatorname{Range} \hat{d}_{i-1} \oplus \operatorname{Ker} \Delta_{i} \oplus$ Range $\hat{\delta}_{i}$. We define the $L^{2}$-cohomology group of $C^{*}$ by $H_{(2)}^{i}\left(C^{*}\right)=\operatorname{Ker} \hat{d}_{i} /$ Range $\hat{d}_{i-1}$. Then, since $\operatorname{Ker} \hat{d}_{i}=\operatorname{Range} \hat{d}_{i-1} \oplus \operatorname{Ker} \Delta_{i}$, we have $\operatorname{Ker} \Delta_{i} \cong$ $H_{(2)}^{i}\left(C^{*}\right)$. Let us investigate the relationship between the $L^{2}$-cohomology and the topological (or, simplicial) homology; see [1, (6.8)].

Corollary 3 (Generalized de Rham pairing). $H_{(2)}^{i}\left(C^{*}\right) \cong\left(I H_{l}^{\bar{m}}(C)\right)^{*}$.

Here $I H_{i}^{\bar{m}}(C)$ means the $i$ th middle intersection homology defined by GoreskyMacPherson. It has the following relation with the usual homology ([4]):

$$
I H_{i}^{\bar{m}}(C) \cong \begin{cases}H_{0}\left(C^{*}\right), & i=0, \\ \operatorname{Image}\left(H_{1}\left(C^{*}\right) \rightarrow H_{1}(C)\right), & i=1, \\ H_{2}(C), & i=2 .\end{cases}
$$

By the usual argument ([10]), to prove Corollary 3 , it suffices to show the following: taking a closed neighborhood $V(\subset C)$ of $S$ and setting $V^{*}=V-S$, we have

$$
H_{(2)}^{i}\left(V^{*}\right) \cong \begin{cases}H_{D R}^{0}(\partial V), & i=0 \\ 0, & i \geq 1 .\end{cases}
$$

In general, the $L^{2}$-cohomology defined by $\left\{\hat{d}_{i}\right\}$ is naturally isomorphic to the one defined by $\left\{d_{i}\right\}$, that is, $\operatorname{Ker} d_{i} / \operatorname{Range} d_{i-1}$. Refer to [1, (1.5)]. Hence let us regard $H_{(2)}^{i}\left(V^{*}\right)$ as to be defined using $\left\{d_{i}\right\}$. Then the isomorphism of (1.3) with $i=0$ will be given by the restriction of smooth functions ( $\supset \operatorname{dom} d_{0}$ ) on $V^{*}$ to its boundary $\partial V$.

Next, paying attention to the Kähler structure of $C^{*}(g)$, let us investigate the pure Hodge structure of its $L^{2}$-cohomology group; see [11]. Denote by $\Lambda^{p, q}\left(C^{*}\right)$ the space of smooth $(p, q)$-forms on $C^{*}$ and define also the space $L^{2} \Lambda^{p, q}\left(C^{*}\right)$ in a similar way. Set $\mathscr{H}_{(2)}^{i}\left(C^{*}\right)=\operatorname{Ker} \Delta_{i}=\left\{\alpha \in L^{2} \Lambda^{i}\left(C^{*}\right) \mid d \alpha=\delta \alpha=0\right\}$ and consider its subspaces $\mathscr{H}_{(2) d}^{p, q}\left(C^{*}\right)=\Lambda^{p, q}\left(C^{*}\right) \cap \mathscr{H}_{(2)}^{p+q}\left(C^{*}\right)$. Moreover we set

$$
H_{(2) d}^{p, q}\left(C^{*}\right)=L^{2} \Lambda^{p, q} \cap \operatorname{Ker} \hat{d}_{p+q} / L^{2} \Lambda^{p, q} \cap \text { Range } \hat{d}_{p+q-1} .
$$

Then we have

Corollary 4 ( $L^{2}$-version of pure Hodge structure).

(1) $\mathscr{H}_{(2)}^{i}\left(C^{*}\right)=\bigoplus_{p+q=i} \mathscr{H}_{(2) d}^{p, q}\left(C^{*}\right), \mathscr{H}_{(2) d}^{p, q}\left(C^{*}\right)=\overline{\mathscr{H}_{(2) d}^{q, p}\left(C^{*}\right)}$.

(2) $\mathscr{H}_{(2) d}^{p, q}\left(C^{*}\right) \cong H_{(2) d}^{p, q}\left(C^{*}\right)$ and hence $H_{(2)}^{i}\left(C^{*}\right) \cong \bigoplus_{p+q=i} H_{(2) d}^{p, q}\left(C^{*}\right)$. 
Notice that we do not use the Dolbeault-type harmonic $L^{2}$-spaces nor $L^{2}$ cohomology groups in the corollary. Once we try to change $\mathscr{H}_{(2) d}^{p, q}\left(C^{*}\right)$ and $H_{(2) d}^{p, q}\left(C^{*}\right)$ into the Dolbeault-type ones, various difficulties arise. We can so far prove only the following assertion for the Dolbeault-type cohomology; compare with Cheeger [2]. We decompose the exterior derivative into $d=\partial+\bar{\partial}$ and its formal adjoint into $\delta=\delta^{\prime}+\delta^{\prime \prime}$ as usual. (The formal adjoint of $\partial$ is denoted by $\delta^{\prime}$.) The operators $\partial_{(p, q)}, \partial_{c,(p, q)}, \hat{\partial}_{(p, q)}, \hat{\partial}_{c,(p, q)}$, etc. are defined in the similar way as $d_{i}, d_{c, i}, \hat{d}_{i}, \hat{d}_{c, i}$, etc. For example, we set $\operatorname{dom} \partial_{(p, q)}=\left\{\alpha \in \Lambda^{p, q}\left(C^{*}\right) \cap L^{2} \Lambda^{p, q} \mid \partial \alpha \in L^{2} \Lambda^{p+1, q}\right\}, \operatorname{dom} \delta_{(p, q)}^{\prime}=\{\alpha \in$ $\left.\Lambda^{p+1, q}\left(C^{*}\right) \cap L^{2} \Lambda^{p+1, q} \mid \delta^{\prime} \alpha \in L^{2} \Lambda^{p, q}\right\}$. Then, denoting by $d V_{g}$ the volume element with respect to our metric $g$, we have

\section{Corollary 5.}

$\operatorname{Ker} \hat{\partial}_{c,(0,0)}=\operatorname{Ker} \hat{\bar{\partial}}_{c,(0,0)}=\operatorname{Ker} \hat{d}_{c, 0}=\operatorname{Ker} \hat{d}_{0}=\left\{\right.$ constant function on $\left.C^{*}\right\}$, $\operatorname{Ker} \hat{\delta}_{c,(0,1)}^{\prime}=\operatorname{Ker} \hat{\delta}_{c,(1,0)}^{\prime \prime}=\operatorname{Ker} \hat{\delta}_{c, 1}=\operatorname{Ker} \hat{\delta}_{1}=\left\{a d V_{g} \mid a \in \mathbf{C}\right\}$.

Finally let us take a nonsingular model $\pi: \widetilde{C} \rightarrow C$ and fix a Kähler metric $d s^{2}$ on $\widetilde{C}$. Then there are three kinds of Kähler manifolds $\widetilde{C}\left(d s^{2}\right), C^{*}\left(d s^{2}\right)$ and $C^{*}(g)$. We will investigate the relationship between their pure Hodge structures.

Corollary 6. (1) The $L^{2}$-cohomology $H_{(2)}^{i}\left(C^{*}\left(d s^{2}\right)\right)$ has also the pure Hodge structure in the sense of Corollary 4.

(2) The restriction map from $\widetilde{C}$ to $C^{*}$ and the identity map on $C^{*}$ induce the isomorphisms

$$
\begin{aligned}
& H_{D R}^{i}(\widetilde{C}) \stackrel{\cong}{\rightrightarrows} H_{(2)}^{i}\left(C^{*}\left(d s^{2}\right)\right) \cong H_{(2)}^{i}\left(C^{*}(g)\right), \quad i \leq 1, \\
& H_{D R}^{2}(\widetilde{C}) \cong H_{(2)}^{2}\left(C^{*}\left(d s^{2}\right)\right) \cong H_{(2)}^{2}\left(C^{*}(g)\right) .
\end{aligned}
$$

(3) The pure Hodge structures for $\widetilde{C}, C^{*}\left(d s^{2}\right), C^{*}(g)$ are all identified through the maps at (2).

Remark. Compare Corollary 6(2), Corollary 3 and (1.2).

\section{Proofs of Corollaries 4-6}

Proof of Corollary 5. Due to the duality, the conjugation and Corollary 1, it suffices to prove $\operatorname{Ker} \hat{\partial}_{c,(0,0)}=\operatorname{Ker} \hat{d}_{c, 0}$. We will prove the nontrivial implication $\operatorname{Ker} \hat{\partial}_{c,(0,0)} \subset \operatorname{Ker} \hat{d}_{c, 0}$. Take $f \in \operatorname{Ker} \hat{\partial}_{c,(0,0)}$. Then there exists a sequence $f_{j} \in \Lambda_{c}^{0}\left(C^{*}\right), j \uparrow \infty$, such that $\lim _{j \rightarrow \infty} f_{j}=f$ and $\lim _{j \rightarrow \infty} \partial f_{j}=0$ (in the $L^{2}$-sense). Using the Hodge identity $\Delta_{d}=2 \Delta_{\partial}$ on $\Lambda_{c}^{0}\left(C^{*}\right)$, we have $\lim _{j \rightarrow \infty}\left(d f_{j}, d f_{j}\right)=\lim _{j \rightarrow \infty}\left(\delta d f_{j}, f_{j}\right)=2 \lim _{j \rightarrow \infty}\left(\delta^{\prime} \partial f_{j}, f_{j}\right)=2 \lim _{j \rightarrow \infty}\left(\partial f_{j}\right.$, $\left.\partial f_{j}\right)=0$. That is, $f$ belongs to $\operatorname{Ker} \hat{d}_{c, 0}$.

Next we will prove Corollary 6(2). For continuous functions $f_{1}$ and $f_{2}$ on some space $Y, f_{1} \lesssim f_{2}$ means that there exists a constant $K>0$ such 
that $f_{1}(y) \leq K f_{2}(y)$ for any $y \in Y$. Moreover we denote by $f_{1} \sim f_{2}$ the situation that both $f_{1} \lesssim f_{2}$ and $f_{1} \gtrsim f_{2}$ hold. These relations are naturally generalized also to the relations between differential forms; for the metrics $g_{1}$ and $g_{2}, g_{1} \sim g_{2}$ precisely means that they are quasi-isometric. Now, on $U-p$, setting $\rho=|u|$ and $\phi=\arg u$, we have $g \sim \rho^{2(m-1)} d \rho^{2}+\rho^{2 m} d \phi^{2}$, $d s^{2} \sim d \rho^{2}+\rho^{2} d \phi^{2}$. Hence the volume elements with respect to $g$ and $d s^{2}$ have the following expressions: $d V_{g} \sim \rho^{2 m-1} d \rho \wedge d \phi, d V_{s} \sim \rho d \rho \wedge d \phi$. We denote the pointwise norms of a form $\alpha$ with respect to $g$ and $d s^{2}$ by $|\alpha|_{g}$ and $|\alpha|_{s}$ respectively. Then, by straightforward computations, we get

Lemma 2.1. On $U-p$, we have $d V_{g} \lesssim d V_{s},|d \rho|_{g}^{2} d V_{g} \sim|d \rho|_{s}^{2} d V_{s},|d \phi|_{g}^{2} d V_{g} \sim$ $|d \phi|_{s}^{2} d V_{s},|d \rho \wedge d \phi|_{g}^{2} d V_{g} \gtrsim|d \rho \wedge d \phi|_{s}^{2} d V_{s}$.

This lemma implies

Corollary 2.2. The identity map on $C^{*}$ induces the following bounded inclusion maps:

$$
\begin{aligned}
L^{2} \Lambda^{i}\left(C^{*}\left(d s^{2}\right)\right) & \rightarrow L^{2} \Lambda^{i}\left(C^{*}(g)\right), & & i \leq 1, \\
L^{2} \Lambda^{i}\left(C^{*}\left(d s^{2}\right)\right) & \leftarrow L^{2} \Lambda^{i}\left(C^{*}(g)\right), & & i \geq 1 .
\end{aligned}
$$

Proof of Corollary 6(2). First we show the isomorphism induced by the identity map on $C^{*}$. Take a closed neighborhood $V(\subset C)$ of $S$ and set $V^{*}=V-S$. Then consider the following commutative diagram consisting of $L^{2}$-versions of long exact sequences for $\left(C^{*}\left(d s^{2}\right), V^{*}\left(d s^{2}\right)\right)$ and $\left(C^{*}(g), V^{*}(g)\right)$ :

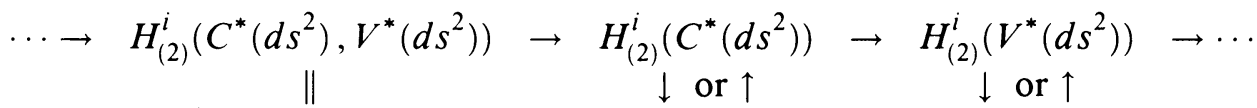

$$
\begin{aligned}
& \cdots \rightarrow \quad H_{(2)}^{i}\left(C^{*}(g), V^{*}(g)\right) \quad \rightarrow \quad H_{(2)}^{i}\left(C^{*}(g)\right) \quad \rightarrow \quad H_{(2)}^{i}\left(V^{*}(g)\right) \quad \rightarrow \cdots
\end{aligned}
$$

Here the maps " $\downarrow$ or $\uparrow$ " mean " $\downarrow$ " if $i \leq 1$ and " $\uparrow$ " if $i=2$ induced by the maps given at Corollary 2.2. Since (1.3) (with $V^{*}=V^{*}(g)$ ) holds also for $V^{*}=V^{*}\left(d s^{2}\right)$, obviously the second " $\downarrow$ or $\uparrow$ " becomes an isomorphism. Hence the five lemma implies that the first " $\downarrow$ or $\uparrow$ " is also an isomorphism. Next let us show the isomorphism induced by the restriction map from $\widetilde{C}$ to $C^{*}$. Due to the same argument as above, $H_{(2)}^{i}\left(\widetilde{C}\left(d s^{2}\right)\right)$ is naturally isomorphic to $H_{(2)}^{i}\left(C^{*}\left(d s^{2}\right)\right)$ (through the restriction), which is a fundamental property of the $L^{2}$-cohomology. Moreover, trivially $H_{D R}^{i}(\tilde{C})$ is isomorphic to $H_{(2)}^{i}\left(\widetilde{C}\left(d s^{2}\right)\right)$.

Next let us prove Corollary 4 . We need some preparations.

Lemma 2.3. On $C^{*}(g)$, we have $\operatorname{dom} \hat{\partial}_{(0,0)} \cap \operatorname{Ker} \hat{\bar{\partial}}_{(0,0)}=\operatorname{Ker} \hat{\partial}_{c,(0,0)}$.

Proof. Refer to Corollaries 1 and 5. Take an element $f$ of the left hand side. Since it belongs to $\operatorname{dom} \hat{d}_{0}=\operatorname{dom} \hat{d}_{c, 0}$, there exists a sequence $f_{j} \in$ $\operatorname{dom} d_{c, 0}=\Lambda_{c}^{0}\left(C^{*}\right)$ such that $\lim _{j \rightarrow \infty} f_{j}=f$, and $\lim _{j \rightarrow \infty} d f_{j}=\hat{d} f$ (in the 
$L^{2}$-sense). Obviously $\hat{d} f=\hat{\partial} f$. Hence $\lim _{j \rightarrow \infty} \bar{\partial} f_{j}=0$. Thus $f$ belongs to $\operatorname{Ker} \hat{\bar{\partial}}_{c,(0,0)}=\operatorname{Ker} \hat{\partial}_{c,(0,0)}$. The converse implication is trivial.

Corollary 2.4. Assume $p+q=1$. Then we have $\mathscr{H}_{(2) d}^{p, q}\left(C^{*}(g)\right)=H_{(2) d}^{p, q}\left(C^{*}(g)\right)$. Proof. Let us study the case $(p, q)=(1,0)$. Since $L^{2} \Lambda^{1,0}\left(C^{*}\right) \cap$ Range $\hat{d}_{0}=$ $\left\{\hat{\partial} f \mid f \in \operatorname{dom} \hat{\partial}_{(0,0)} \cap \operatorname{Ker} \hat{\bar{\partial}}_{(0,0)}\right\}$, Lemma 2.3 implies that it is equal to $\{0\}$. Hence $H_{(2) d}^{1,0}\left(C^{*}(g)\right)=L^{2} \Lambda^{1,0}\left(C^{*}\right) \cap \operatorname{Ker} \hat{d}_{1}$. And its element $\varphi$ satisfies $\delta \varphi=$ $-\bar{*}_{g} d \bar{*}_{g} \varphi=-\sqrt{-1} \overline{1}_{g} d\left(\bar{\varphi} \wedge \omega_{g}\right)=0$, where $\bar{*}_{g}$ and $\omega_{g}$ are the complex star operator and the Kähler form associated to our Kähler metric $g$. Thus the proof is complete.

Proof of Corollary 4. Consider the following commutative diagram.

$$
\begin{aligned}
& \bigoplus_{p+q=1} \mathscr{H}_{(2) d}^{p, q}\left(C^{*}(g)\right) \quad \overrightarrow{\mathscr{H}} \mathscr{H}_{(2)}^{1}\left(C^{*}(g)\right) \\
& \gamma_{p, q} \| \quad \gamma_{1} \downarrow \cong \\
& \bigoplus_{p+q=1} H_{(2) d}^{p, q}\left(C^{*}(g)\right) \quad \rightarrow \quad H_{(2)}^{1}\left(C^{*}(g)\right) \\
& \alpha \uparrow \quad \beta \uparrow \cong \\
& \bigoplus_{p+q=1} H^{p, q}(\widetilde{C}) \quad \underset{\vec{i}}{\cong} \quad H_{D R}^{1}(\widetilde{C})
\end{aligned}
$$

Here $H^{p, q}(\widetilde{C})$ is the $(p, q)$-Dolbeault cohomology group of $\widetilde{C}$ and the isomorphism $\tilde{l}$ is given by the pure Hodge structure of $H_{D R}^{2}(\widetilde{C})$. The isomorphisms $\beta$ and $\gamma_{1}$ have already been shown in Corollary 6(2) and the argument preceeding Corollary 3 in $\S 1$. Moreover the identity $\gamma_{p, q}$ has been given in Corollary 2.4. and obviously the map $l_{\mathscr{L}}$ is injective. Hence all the maps $l_{\mathscr{H}}, l, \alpha$ are easily proved to be isomorphic.

Lastly we have to prove (1) and (3) of Corollary 6, which may be obvious now. That is, Lemma 2.3 and Corollary 2.4 hold also for $C^{*}\left(d s^{2}\right)$ and we have also the commutative diagram (2.1) with $g$ replaced by $d s^{2}$. Hence we have (1). The assertion (3) can be shown by the commutative diagrams for $C^{*}(g)$ and $C^{*}\left(d s^{2}\right)$.

\section{ACKNOWLEDGMENT}

Finally the author would like to thank the referee for invaluable advice and for informing him about the paper [5].

\section{REFERENCES}

1. J. Cheeger, On the Hodge theory of Riemannian pseudomanifolds, Proc. Symp. Pure Math. 36, Providence, RI, 1980, 91-146.

2. __ Hodge theory of complex cones, Astérisque 101-102 (1983), 118-134.

3. __ Spectral geometry of singular Riemannian spaces, J. Diff. Geom. 18 (1983), 575-657.

4. J. Cheeger, M. Goresky and R. MacPherson, $L^{2}$-cohomology and intersection homology for singular algebraic varieties, Seminar on Differential Geometry, Ann. Math. Studies 102, 1982, 303-340. 
5. P. Haskell, Index theory of curves, Trans. Amer. Math. Soc. 288 (1985), 591-604.

6. W. C. Hsiang and V. Pati, $L^{2}$-cohomology of normal algebraic surfaces I, Invent. Math. 81 (1985), 395-412.

7. M. Nagase, The fundamental solutions of the heat equations on Riemannian spaces with conelike singular points, Kodai Math. J. 7 (1984), 382-455.

8. _ On the heat operators of cuspidally stratified Riemannian spaces, Proc. Japan Acad., Ser. A 62 (1986), 58-60.

9. _ On the heat operators of normal singular algebraic surfaces, J. Differential Geom. 28 (1988), 37-57.

10. _ Remarks on the $L^{2}$-cohomology of singular algebraic surfaces, J. Math. Soc. Japan 41 (1989), 97-116.

11. _ Pure Hodge structure of the harmonic $L^{2}$-forms on singular algebraic surfaces, Publ. RIMS Kyoto Univ. 24 (1988), 1005-1023.

12. M. Namba, Geometry of projective algebraic curves, Marcel Dekker, New York and Basel, 1984.

Department of Mathematics, Tokyo Institute of Technology, Oh-OKayama, MeguroKU, TOKYO 152, JAPAN

Current address: Department of Mathematics, Faculty of Science, Saitama University, Urawa, Saitama 338, Japan 